\title{
(6) OPEN ACCESS \\ Impact of environmental tobacco smoke exposure on anaesthetic and surgical outcomes in children: a systematic review and meta-analysis
}

\author{
Christopher Chiswell, ${ }^{1}$ Yasmin Akram $^{2}$
}

\begin{abstract}
- Additional material is published online only. To view please visit the journal online (http://dx.doi.org/10.1136/ archdischild-2016-310687).

${ }^{1}$ Department of Public Health, Birmingham Children's Hospital, Birmingham, UK ${ }^{2}$ Institute of Applied Health Research, University of Birmingham, Birmingham, UK
\end{abstract}

Correspondence to Dr Christopher Chiswell, Department of Public Health, Birmingham Children's Hospital, Steelhouse Lane, Birmingham B4 6NH, UK; Christopher.chiswell@bch.nhs.uk

Received 11 February 2016 Revised 8 June 2016 Accepted 20 June 2016 Published Online First 14 July 2016

\section{SLinked}

- http://dx.doi.org/10.1136/ archdischild-2016-311652

\section{CrossMark}

To cite: Chiswell $C$ Akram Y. Arch Dis Child 2017;102:123-130.

\section{ABSTRACT}

Background Tobacco smoke exposure in adults is linked to adverse anaesthetic and surgical outcomes. Environmental tobacco smoke (ETS) exposure, including passive smoking, causes a number of known harms in children, but there is no established evidence review on its impact on intraoperative and postoperative outcomes. Objectives To undertake a systematic review of the impact of ETS on the paediatric surgical pathway and to establish if there is evidence of anaesthetic,

intraoperative and postoperative harm.

Eligibility criteria participants Children aged 0-18 years undergoing anaesthetic or surgical procedures, any country, English language papers. Exposure ETS exposure assessed via questioning, observation or biological marker.

Outcome measures Frequency of respiratory and other adverse events during anaesthesia, surgery and recovery, and longer term surgical outcomes.

Results 28 relevant studies were identified; 15 considered anaesthetic outcomes, 12 surgical outcomes, and 1 a secondary outcome. There was sufficient evidence to demonstrate that environmental smoke exposure significantly increased risk of perianaesthetic respiratory adverse events (Pooled risk ratio $2.52 \mathrm{Cl} 95 \%$ 1.68 to 3.77$)$, and some evidence that ear and sinus surgery outcomes were poorer for children exposed to ETS.

Conclusions ETS exposure increases the risk of anaesthetic complications and some negative surgical outcomes in children, and this should be considered when planning surgery. Research is required to demonstrate whether changes in household smoking behaviour prior to surgery reduces risk of adverse outcomes, and to close the evidence gap around other outcomes such as wound healing and respiratory infections.

Trial registration number Review registration number 42014014557.

\section{INTRODUCTION}

Environmental tobacco smoke (ETS) exposure, also referred to as 'secondhand smoke' or 'passive smoking', is exposure to smoke from the burning of a tobacco product and smoke that is exhaled by smokers. ETS exposure has been demonstrated to have a significant impact on the health of children and young people. This includes increased incidence of respiratory infection, ${ }^{1}$ asthma, ${ }^{2}$ ear infection ${ }^{3}$ and meningitis. ${ }^{4}$ Children are more vulnerable than adults to ETS, ${ }^{5}$ and there is consensus on the importance of protecting children from the harms of ETS exposure. ${ }^{6}$

\section{What is already known on this topic?}

Environmental tobacco smoke exposure has a significant impact on paediatric health, including frequency of respiratory illness, bacterial meningitis and ear infections.

- Smoking by adults increases their risk of anaesthetic and surgical complications, including delayed wound healing, increased respiratory complications and delayed discharge.

- Appropriate preoperative smoking cessation in adults reduces the risk of these complications.

\section{What this study adds?}

- This first review of the evidence identifies and summarises the effect of environmental tobacco smoke exposure on paediatric anaesthetic and surgical outcomes.

- A pooled estimate suggests that environmental tobacco smoke exposure significantly increases risk of paediatric respiratory adverse events during the perianaesthetic period.

- There is a need to establish whether household health promotion interventions and preoperative smoking cessation reduce the risk of harmful outcomes for children undergoing surgery.

Rationale

In adults, there is evidence that tobacco smoking has a negative impact on surgical outcomes, including anaesthetic complications, ${ }^{7}$ reduced wound and bone healing ${ }^{8} 9$ and increased morbidity and delayed discharge from hospital. ${ }^{10}$ Appropriate cessation of smoking before an operative episode reduces the likelihood of these events and improves patient outcomes. ${ }^{11}$ Economic benefits of effective preoperative smoking cessation have been modelled in adults, where cessation was conservatively estimated to save between one-half and three-quarters of a million pounds in London alone, with a potential to extend this to $5-13$ million pounds with a more engaged programme. ${ }^{12}$

There is no similar evidence base around the impact of ETS exposure levels on surgery in children, and a review of PROSPERO abstracts suggests no similar systematic reviews (SRs) are in preparation. 


\section{Objectives}

The objective of this SR was to answer the structured question 'What is the evidence from observational studies that environmental tobacco smoke (ETS) exposure in children aged under 18 affects anaesthetic and surgical outcomes compared with those with lower or no ETS exposure?'

\section{METHODS}

This SR was conducted in line with current PRISMA guidance. Review coauthors agreed the review protocol, which was submitted to the University of York PROSPERO International Prospective Register of Systematic Reviews (ID 42014014557).

\section{Eligibility criteria}

Studies were eligible if they met the following four criteria:

(A) An observational study (as defined by the Cochrane Collaboration), including case-control, cohort, case-cohort, nested case-control and cross-sectional studies. Case studies, letters, editorials and reviews were excluded. Where relevant cohorts could be identified as nested within an interventional study (eg, a randomised controlled trial collecting ETS exposure as confounding), it was included.

(B) A population of children aged 18 or under undergoing any operative procedures, including studies of children, or containing a subgroup of children aged 18 or under.

(C) Study or subgroup analysis contained within it uses reported or observed ETS (eg, by parental questionnaire), or biological markers thereof, as an independent variable on outcome.

(D) Study included either any anaesthetic, recovery and surgical outcomes, for our protocol's primary outcomes, or secondary outcomes including length of stay, cost of care, patient experience or change in close contact smoking behaviours or ETS exposure. Any length of follow-up was accepted.

\section{Information sources}

A standardised search protocol was developed and performed across all databases on 30 October 2014. We searched only English language publications.

MEDLINE (1946-2014), Embase (1974-2014) and PsycINFO (1967-2014) were searched via OVID, as well as IBSS (1951-2014) via ProQuest, CPCI (1990-2013) via Web of Science, CINAHL Plus (1964-2014) via EBSCO Health and OpenGray (inception-2014). The sample search strategy for MEDLINE is available in the online supplementary file S1. Reference lists of studies selected for inclusion were reviewed, but no further papers were identified. No contacts were made with authors to identify or obtain papers. Search results were imported into Mendeley (v1.12.3) and de-duplicated algorithmically, followed by manual inspection. A check was performed on final papers to confirm no duplicated publications.

\section{Study selection}

Two reviewers (CC and YA) independently screened titles and abstracts against the eligibility criteria. Where there was no consensus, the reviewers engaged in dialogue, with unresolved papers taken forward to full review. Full papers were obtained for all abstracts taken forward, and reviewed independently by the two reviewers against a pre-established checklist. Again, consensus on inclusion was reached through dialogue. A third reviewer was available for resolution of disagreements, but was not required.

\section{Data extraction}

A data extraction template was created to capture participant numbers, specialty, inclusion and exclusion criteria, measurement of ETS exposure, whether ETS was a primary or secondary measure and how ETS exposure was categorised. Outcomes were captured, including type of outcome, length of study, statistical method, results and adjustments for bias and confounding. Where only rates or percentages were reported, these were used to calculate raw numbers for inclusion.

Wherever possible, total and included study participants, raw event numbers and summary statistics were extracted. Other specific outcomes, such as 'time to event' were captured narratively. Primary outcomes were classified into three categories: anaesthetic (eg, laryngospasm), immediate postsurgical (eg, bleeding) and long-term surgical (eg, disease resolution or improvement).

The extraction tool was piloted on a scoping study, and then applied consistently to all papers by a study reviewer (YA). Results were independently cross-checked by the second reviewer (CC).

\section{Quality scoring}

Studies that met the inclusion criteria were independently scored for methodological quality by the two reviewers using a modified eight-point version of the Newcastle-Ottawa quality assessment scale (http://www.ohri.ca/programs/clinical_epidemiology/ oxford.asp) recognised by the Cochrane Collaboration Non-Randomised Studies Working Group. The content validity and inter-rater reliability of the tool are already established. The study reviewers selected a score of 7 or more as an indicator of high methodological quality.

\section{Statistical methods}

The protocol described that quantitative data synthesis would be undertaken where there were sufficient data. Surgical outcomes were too diverse, and are reported narratively. Anaesthetic respiratory adverse events (RAEs) were reported more consistently. A pooled, weighted risk ratio was estimated using random effects meta-analysis performed in RevMan V.5.3.5, ${ }^{13}$ using the Mantel-Haenszel implementation of the DerSimonian Laird model, accompanied by tests for heterogeneity ( $\mathrm{I}^{2}$ statistic). A random effects model was chosen to reflect the range of variation in participants and specialties, and to support the understanding of impacts of ETS exposure on wider surgical populations.

The analysis used available data from all papers which measured ETS exposure as a primary variable of interest, and reported RAE outcomes. Where RAEs were reported in a way that could not be combined, laryngospasm was chosen as the dominant proxy to take into the meta-analysis. Where several time points were reported, the first time point after administration of anaesthetic was selected for inclusion, as it represented the theoretical point of maximum impact of ETS on RAEs.

The impact of missing data was not examined due to limitations in the available datasets and the heterogeneous nature of the studies. Sensitivity was tested by removing individual studies, and then the two most heavily weighted studies, from our analyses.

\section{RESULTS}

\section{Study selection}

Of the 6875 titles assessed for eligibility, 48 proceeded to full text review, with 28 studies fitting the full eligibility criteria. Figure 1 summarises the selection process. Twenty-four studies ${ }^{14-37}$ used cohorts to examine ETS exposure, and four ${ }^{38-41}$ used casecontrol methodologies. 


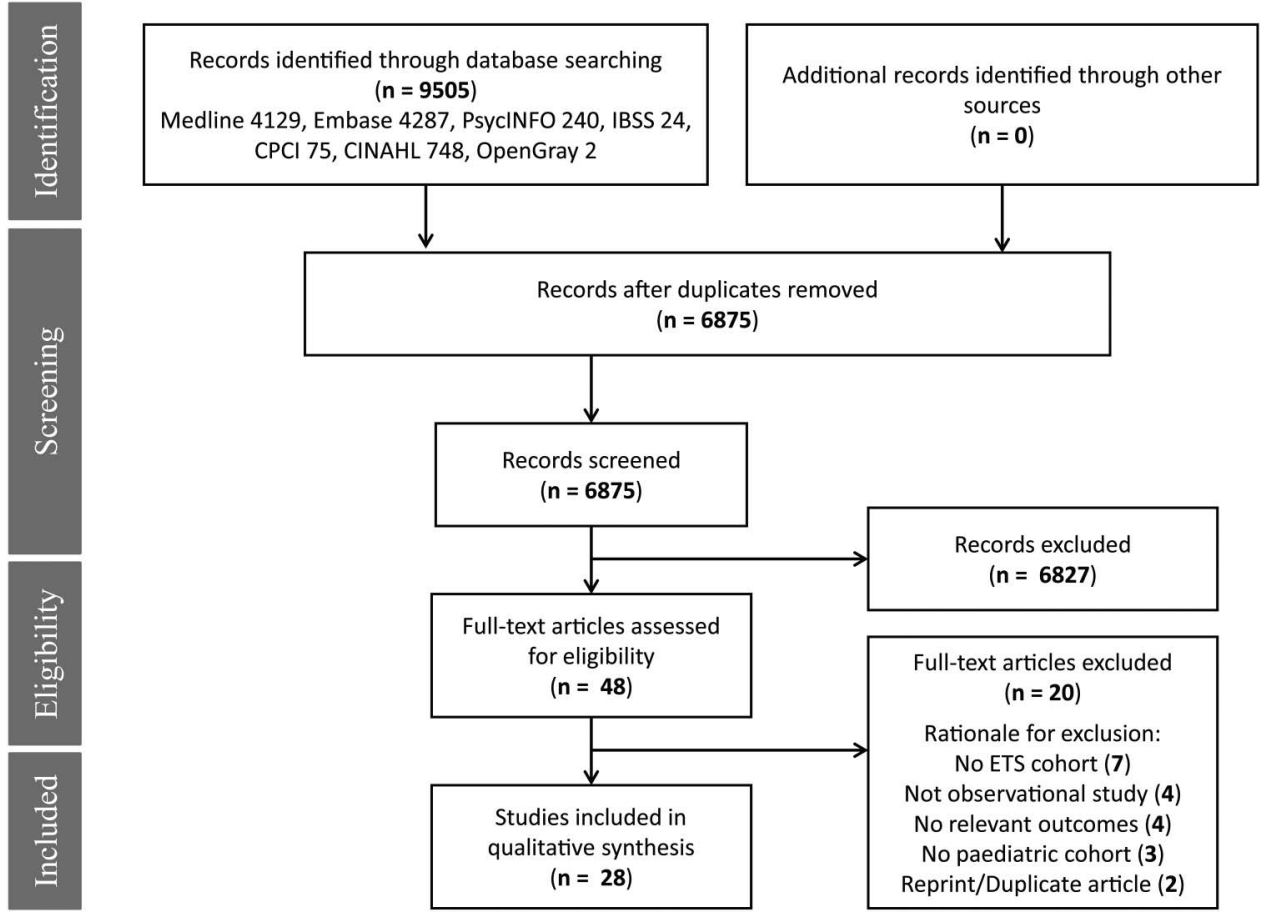

Figure 1 Flow chart of the inclusion and exclusion processes for the systematic review. ETS, environmental tobacco smoke.

\section{Study characteristics}

Fifteen studies considered anaesthetic outcomes, ${ }^{14-27} 41$ and twelve studies considered surgical outcomes. ${ }^{28-34} 36$ 38-40 One further study ${ }^{37}$ considered behaviour change, a secondary outcome. No studies identified immediate surgical outcomes and, unlike the adult evidence base, no studies considered longterm outcomes of wound or bone healing.

Studies covered ages from birth upwards. One study ${ }^{41}$ had a protocol that focused on children, but which reported an age range of $0-22$ years in its data. The mean age was 5.8 , and the reviewers agreed the study should be included as the majority of patients were within the protocol.

The anaesthetic outcome studies used a wide range of surgical groups, including general or mixed surgery, ${ }^{15}$ 18-20 $22-24 \quad 2741$ dental, ${ }^{25}$ ophthalmology ${ }^{16}$ and urology ${ }^{17}$ patients.

All 12 studies considering surgical outcomes focused on ear, nose and throat (ENT) patients. The impact of ETS on this patient group was complicated by the confounding associated with ETS being a cause of delayed surgical recovery and a cause of primary disease or recurrence. In five studies, ${ }^{29} 31-3338$ ETS exposure was treated as a confounding variable, rather than the primary focus of the study.

ETS exposure was usually measured as a binary exposed/ unexposed variable, although additional measures such as identifying which family members smoked, ${ }^{17}{ }^{20}$ levels of smoking ${ }^{29}$ and biological markers ${ }^{14} 23$ were also used. One study showed good correlation between parental questioning and urinary cotinine levels. ${ }^{14}$

Studies reported a range of biological and clinical outcomes; the majority of anaesthetic outcomes reported were RAEs, particularly laryngospasm. Oxygen desaturations $\begin{array}{llll}18 & 20 & 22 & 27\end{array}$ and muscle relaxant dosages ${ }^{21}$ were used as biological measures. Long-term surgical outcome markers included ciliary regeneration, ${ }^{30}$ as well as clinical measures such as change in symptoms and signs, ${ }^{34} 3639$ complications ${ }^{32} 3540$ or repeat procedures. ${ }^{34} 38$

\section{Settings}

Studies from Australia, Canada, Finland, Ireland, South Korea, Switzerland, Turkey, UK and USA were included.

\section{Risk of bias}

Of the 15 studies considering anaesthetic outcomes, 10 were assessed as high quality; for the 12 considering ENT surgical outcomes, 2 were assessed as high quality. The majority of surgical studies considered ETS exposure as a confounding factor in a wider intervention study, and the ETS impact related only to specific subcohorts which had been given less methodological consideration. Online supplementary file S2 contains a summary of the assessment.

Table 1 summarises the characteristics of the studies examining anaesthetic and recovery outcomes, including methodology, age groups and sample sizes and measurement technique for ETS. It also summarises relevant outcome measures. A similar table describing the surgical papers is available in the online supplementary file S3.

\section{Individual study outcomes}

Anaesthetic outcomes

Table 2 describes the key findings of studies related to anaesthetic outcomes. Three of fifteen studies ${ }^{18} 1925$ considering anaesthetic outcomes did not show that ETS had significant impact on anaesthetic outcomes, with a further study ${ }^{26}$ showing a higher rate of RAE but not undertaking a statistical analysis. The remaining 11 studies showed significant effects. The most common adverse outcomes were increased rates of laryngospasm, bronchospasm and coughing. Both studies considering biological markers ${ }^{2021}$ demonstrated some impact of ETS exposure.

Pooled estimate of respiratory adverse event (RAE) risk

Eleven studies ${ }^{14-18} 20222325-27$ were eligible for dichotomous meta-analysis with a random effects model to create a pooled 
Table 1 Characteristics of 15 studies focusing on anaesthetic and recovery outcomes

\begin{tabular}{|c|c|c|c|c|c|c|c|c|c|}
\hline Study author & Year & $\begin{array}{l}\text { Outcome } \\
\text { methodology }\end{array}$ & $\begin{array}{l}\text { Age group } \\
\text { (identified/ } \\
\text { included) }\end{array}$ & Surgical group & Location & $\begin{array}{l}\text { ETS measure } \\
\text { (number exposed) }\end{array}$ & $\begin{array}{l}\text { ETS } \\
\text { priority }\end{array}$ & Outcomes & $\begin{array}{l}\text { Quality } \\
\text { score }\end{array}$ \\
\hline Drongowski ${ }^{14}$ & 2003 & $\begin{array}{l}\text { Prospective } \\
\text { cohort }\end{array}$ & $\begin{array}{l}3 \text { months- } \\
13 \text { years } \\
(n=146 / 146)\end{array}$ & $\begin{array}{l}\text { Inguinal hernia } \\
\text { repair }\end{array}$ & USA & $\begin{array}{l}\text { Parent } \\
\text { questionnaire on } \\
\text { smoking history/ } \\
\text { exposure and } \\
\text { urinary cotinine } \\
\text { (57 ETS+) }\end{array}$ & $1^{\circ}$ & $\begin{array}{l}\text { Observed respiratory } \\
\text { events in induction, } \\
\text { emergence and recovery }\end{array}$ & 8 \\
\hline Jones $^{15}$ & 2006 & $\begin{array}{l}\text { Prospective } \\
\text { cohort }\end{array}$ & $\begin{array}{l}1-18 \text { years } \\
(n=428 / 405)\end{array}$ & $\begin{array}{l}\text { Elective day case: } \\
\text { mixed specialties }\end{array}$ & USA & $\begin{array}{l}\text { Parent completed } \\
\text { risk factor } \\
\text { questionnaire, } \\
\text { included smoking } \\
\text { history (168 ETS+) }\end{array}$ & $1^{\circ}$ & $\begin{array}{l}\text { Airway complications } \\
\text { observed against graded } \\
\text { scale intraoperatively } \\
\text { and post anaesthesia }\end{array}$ & 7 \\
\hline $\mathrm{Kim}^{16}$ & 2013 & $\begin{array}{l}\text { Retrospective } \\
\text { cohort }\end{array}$ & $\begin{array}{l}\text { Child: not } \\
\text { defined } \\
(n=159 / 159)\end{array}$ & $\begin{array}{l}\text { Had URI } \\
\text { symptom; } \\
\text { ENT and } \\
\text { ophthalmology }\end{array}$ & $\begin{array}{l}\text { South } \\
\text { Korea }\end{array}$ & $\begin{array}{l}\text { Passive smoking } \\
\text { documented in } \\
\text { anaesthetic record } \\
\text { (28 ETS+) }\end{array}$ & $1^{\circ}$ & $\begin{array}{l}\text { Respiratory adverse } \\
\text { events documented in } \\
\text { notes at intubation, } \\
\text { intra-extubation and } \\
\text { post-extubation, and in } \\
\text { recovery }\end{array}$ & 7 \\
\hline Lakshmipathy $^{17}$ & 1996 & $\begin{array}{l}\text { Retrospective } \\
\text { cohort }\end{array}$ & $\begin{array}{l}\text { Child: not } \\
\text { defined } \\
(n=322 / 310)\end{array}$ & $\begin{array}{l}\text { Elective day case: } \\
\text { ENT and urology }\end{array}$ & USA & $\begin{array}{l}\text { Parent reported } \\
\text { regular smoker at } \\
\text { home ( } 96 \mathrm{ETS}+)\end{array}$ & $1^{\circ}$ & $\begin{array}{l}\text { Laryngospasm } \\
\text { documented in records }\end{array}$ & 7 \\
\hline Lyons $^{18}$ & 1996 & $\begin{array}{l}\text { Prospective } \\
\text { cohort }\end{array}$ & $\begin{array}{l}1-12 \text { years } \\
(n=? / 125)\end{array}$ & $\begin{array}{l}\text { Elective day case: } \\
\text { not ENT }\end{array}$ & Ireland & $\begin{array}{l}\text { Parental } \\
\text { questionnaire of } \\
\text { home and other } \\
\text { smoker contacts ( } 63 \\
\text { ETS+) }\end{array}$ & $1^{\circ}$ & $\begin{array}{l}\text { Respiratory adverse } \\
\text { events observed by } \\
\text { blinded anaesthetist at } \\
\text { preoperative, } \\
\text { intraoperative and } \\
\text { postoperative points }\end{array}$ & 6 \\
\hline Mamie $^{19}$ & 2004 & $\begin{array}{l}\text { Prospective } \\
\text { cohort }\end{array}$ & $\begin{array}{l}1-14 \text { years } \\
(n=800 / 743)\end{array}$ & Mixed specialties & Switzerland & $\begin{array}{l}\text { Parent completed } \\
\text { allergy/ asthma } \\
\text { questionnaire, } \\
\text { including smoking } \\
\text { (429 ETS+) }\end{array}$ & $2^{\circ}$ & $\begin{array}{l}\text { Intraoperative, } \\
\text { postoperative and } \\
\text { perioperative respiratory } \\
\text { adverse events }\end{array}$ & 7 \\
\hline $0^{\prime}$ Rourke $^{20}$ & 2006 & $\begin{array}{l}\text { Prospective } \\
\text { matched } \\
\text { cohort }\end{array}$ & $\begin{array}{l}5-15 \text { years } \\
\text { ( } n=93 \text { at } \\
\text { recovery; } 96 \text { at } \\
\text { discharge) }\end{array}$ & $\begin{array}{l}\text { Convenience } \\
\text { sample; } \\
\text { non-cavity } \\
\text { surgery }\end{array}$ & USA & $\begin{array}{l}\text { Direct parental } \\
\text { questioning ( } 46 \text { ETS } \\
+ \text { at recovery, } 49 \\
\text { ETS+ at discharge) }\end{array}$ & $1^{\circ}$ & $\begin{array}{l}\text { Pulmonary function as } \\
\text { percentage predicted; } \\
\text { respiratory events } \\
\text { secondary outcome }\end{array}$ & 7 \\
\hline Parnis $^{41}$ & 2011 & $\begin{array}{l}\text { Prospective } \\
\text { case-control }\end{array}$ & $\begin{array}{l}\text { Child: not } \\
\text { defined* }^{*} \\
(n=? / 2051)\end{array}$ & Mixed specialties & Australia & $\begin{array}{l}\text { Parental smoking } \\
\text { status as part of } \\
\text { wider research } \\
\text { questionnaire ( } 926 \\
\text { ETS+) }\end{array}$ & $2^{\circ}$ & $\begin{array}{l}\text { 'Good' vs 'bad' } \\
\text { anaesthetic outcome, } \\
\text { including adverse } \\
\text { respiratory, } \\
\text { gastrointestinal and } \\
\text { circulatory events }\end{array}$ & 6 \\
\hline Reisli $^{21}$ & 2004 & $\begin{array}{l}\text { Prospective } \\
\text { cohort }\end{array}$ & $\begin{array}{l}4-10 \text { years } \\
(n=40 / 40)\end{array}$ & $\begin{array}{l}\text { ENT surgery; } \\
\text { convenience } \\
\text { sample }\end{array}$ & Turkey & $\begin{array}{l}\text { Parental } \\
\text { questioning of } \\
\text { child's overall ETS } \\
\text { exposure ( } 20 \text { ETS+) }\end{array}$ & $1^{\circ}$ & $\begin{array}{l}\text { Rocuronium dose } \\
\text { required for } \\
\text { neuromuscular blockade }\end{array}$ & 6 \\
\hline Seyidov $^{22}$ & 2011 & $\begin{array}{l}\text { Prospective } \\
\text { cohort }\end{array}$ & $\begin{array}{l}3 \text { months- } \\
12 \text { years } \\
(n=402+/ 385)\end{array}$ & Elective surgery & Turkey & $\begin{array}{l}\text { Parent questioning } \\
\text { of quantity of } \\
\text { smoke exposure } \\
\text { (234 ETS+) }\end{array}$ & $1^{\circ}$ & $\begin{array}{l}\text { Respiratory adverse } \\
\text { events observed by } \\
\text { blinded team }\end{array}$ & 7 \\
\hline Skolnick $^{23}$ & 1998 & $\begin{array}{l}\text { Prospective } \\
\text { cohort }\end{array}$ & $\begin{array}{l}1 \text { month- } \\
12 \text { years } \\
(n=602 / 499)\end{array}$ & Elective surgery & USA & $\begin{array}{l}\text { Urinary cotinine } \\
\text { and parental } \\
\text { enquiry ( } 262 \mathrm{ETS}+)\end{array}$ & $1^{\circ}$ & $\begin{array}{l}\text { Respiratory adverse } \\
\text { events }\end{array}$ & 8 \\
\hline Tait $^{24}$ & 2001 & $\begin{array}{l}\text { Prospective } \\
\text { cohort }\end{array}$ & $\begin{array}{l}1 \text { month- } \\
18 \text { years } \\
(n=1078 / 407)\end{array}$ & $\begin{array}{l}\text { Elective surgery } \\
\text { with active URI }\end{array}$ & USA & $\begin{array}{l}\text { Questionnaire } \\
\text { including parental } \\
\text { smoking habits }\end{array}$ & $2^{\circ}$ & $\begin{array}{l}\text { Contribution of factor to } \\
\text { overall predictive } \\
\text { multiple regression } \\
\text { model }\end{array}$ & 6 \\
\hline Thikkurissy $^{25}$ & 2012 & $\begin{array}{l}\text { Prospective } \\
\text { cohort }\end{array}$ & $\begin{array}{l}19 \text { months- } \\
12 \text { years ( } \mathrm{n}=\text { ?/ } \\
99)\end{array}$ & Dental & USA & $\begin{array}{l}\text { Parental interview } \\
\text { on smoking status } \\
\text { and quantity } \\
\text { (51 ETS+) }\end{array}$ & $1^{\circ}$ & $\begin{array}{l}\text { Respiratory adverse } \\
\text { events observed by } \\
\text { blinded anaesthetist and } \\
\text { recovery nurse }\end{array}$ & 7 \\
\hline Tütüncü $^{26}$ & 2011 & $\begin{array}{l}\text { Prospective } \\
\text { cohort }\end{array}$ & $\begin{array}{l}1-15 \text { years } \\
(\mathrm{n}=? / 150)\end{array}$ & $\begin{array}{l}\text { Convenience } \\
\text { sample; lower } \\
\text { abdominal/ } \\
\text { urology }\end{array}$ & Turkey & $\begin{array}{l}\text { Parental } \\
\text { questionnaire } \\
\text { including parent } \\
\text { smoking status } \\
\text { (100 ETS+) }\end{array}$ & $1^{\circ}$ & $\begin{array}{l}\text { Secondary outcome of } \\
\text { postoperative respiratory } \\
\text { complications }\end{array}$ & 5 \\
\hline $\begin{array}{l}\text { von } \\
\text { Ungern-Sternberg } \\
\text { et } a 2^{27}\end{array}$ & 2010 & $\begin{array}{l}\text { Prospective } \\
\text { cohort }\end{array}$ & $\begin{array}{l}\text { Child: not } \\
\text { defined } \\
(n=10496 / \\
9038)\end{array}$ & $\begin{array}{l}\text { Anaesthesia for } \\
\text { elective and } \\
\text { urgent }\end{array}$ & Australia & $\begin{array}{l}\text { Parent smoking } \\
\text { report at } \\
\text { preoperation check } \\
(2929 \text { ETS+) }\end{array}$ & $1^{\circ}$ & $\begin{array}{l}\text { Perioperative adverse } \\
\text { events }\end{array}$ & 8 \\
\hline
\end{tabular}

\footnotetext{
*Study age range from 0 to 22 years. Mean age 5.8. Reviewers made decision to include in study.
}

tThere is an apparent numerical error in the original paper where the identified total appears not to include exclusions.

ENT, ear, nose and throat; ETS, environmental tobacco smoke; URI, upper respiratory infection. 
Table 2 Key anaesthetic outcomes relevant to the protocol

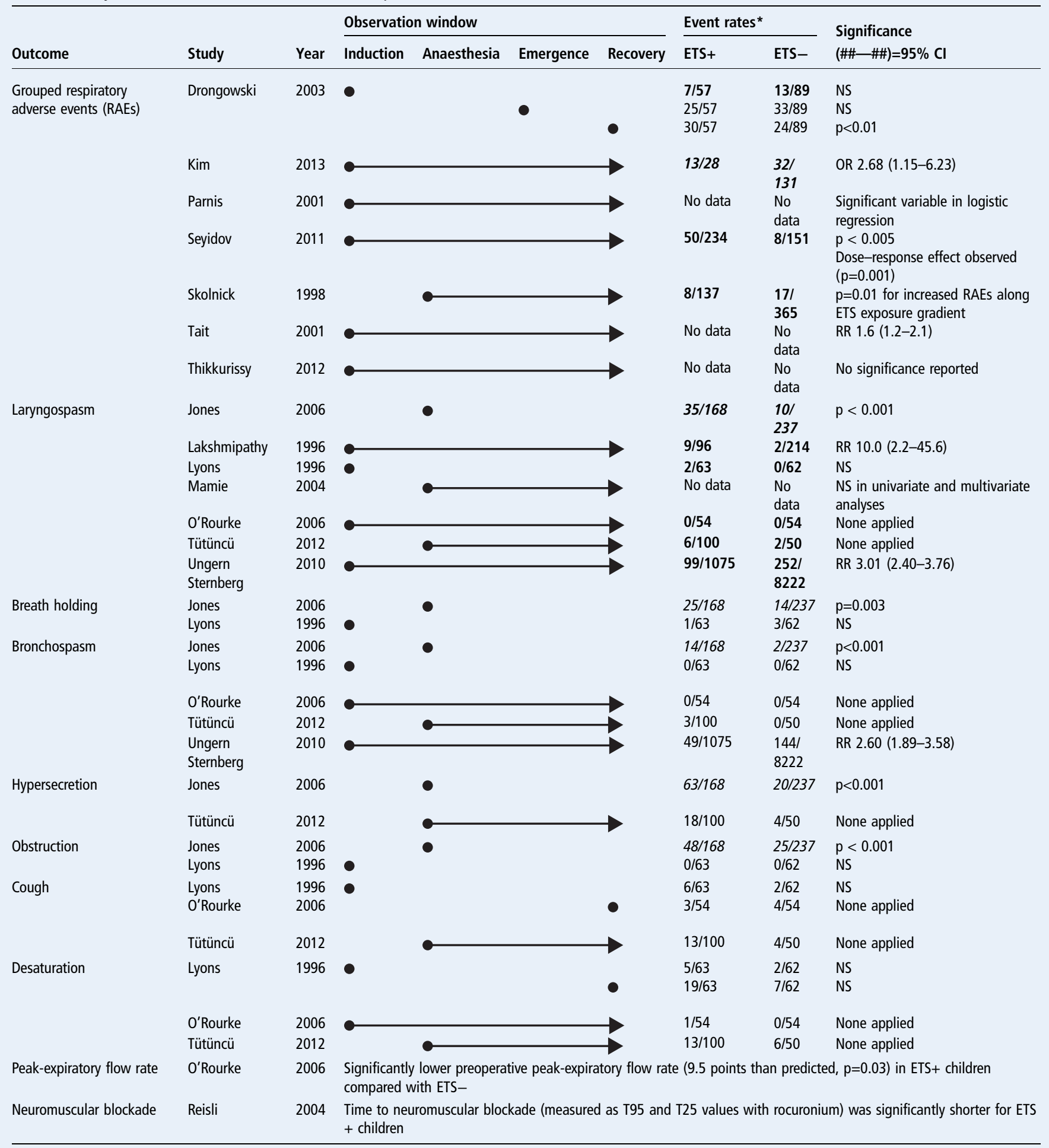

*Italic data inferred where only percentages available; bold data selected for inclusion in systematic review.

$\mathrm{Cl}$, confidence interval; ETS, environmental tobacco smoke; NS, non-significant; OR, odds ratio; RR, relative risk.

estimate of the risk ratio of a RAE with ETS exposure. Data were not available for one study, ${ }^{25}$ and attempts to contact the author were unsuccessful.

Figure 2 shows the outcome of the analysis, demonstrating that ETS exposure showed an increased risk ratio for a RAE in the perianaesthetic period of 2.52 (95\% CI 1.68 to 3.77 , $\mathrm{p}<0.0001)$ compared with not being exposed. There was moderate statistical heterogeneity $\left(\mathrm{I}^{2}=62 \%\right)$. Subgroup analysis of studies focusing only on laryngospasm was undertaken, recognising the variety of outcomes that grouped RAEs would assess. This retained significance with a risk ratio of 3.54 (95\% CI 2.37 to $5.28, \mathrm{p}<0.001)$ and reduced heterogeneity $\left(\mathrm{I}^{2}=23 \%\right)$. 


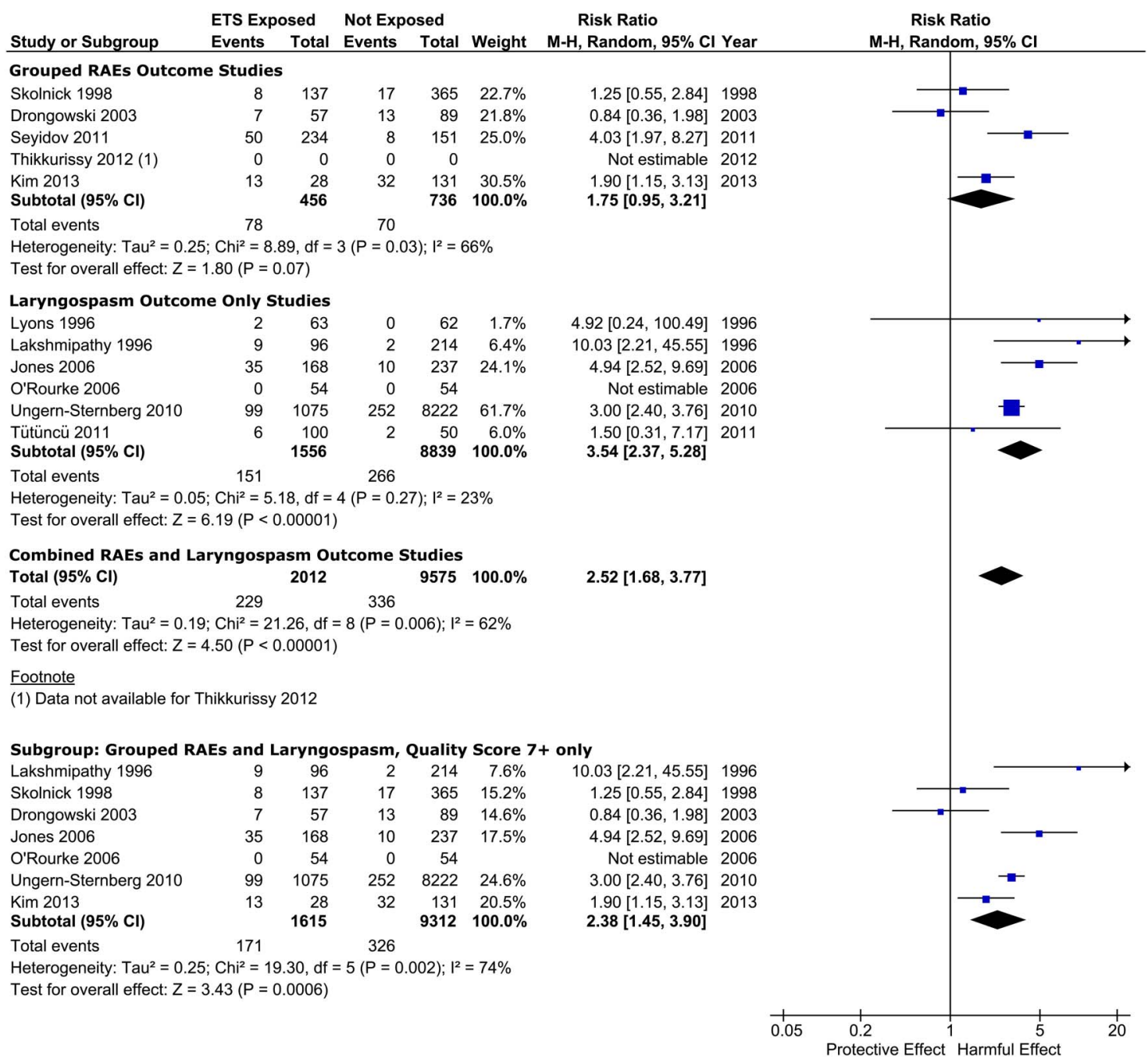

Figure 2 Meta-analysis of environmental tobacco smoke (ETS) exposure and its association with likelihood of respiratory adverse events (RAEs) in the perianaesthetic period, including subgroups.

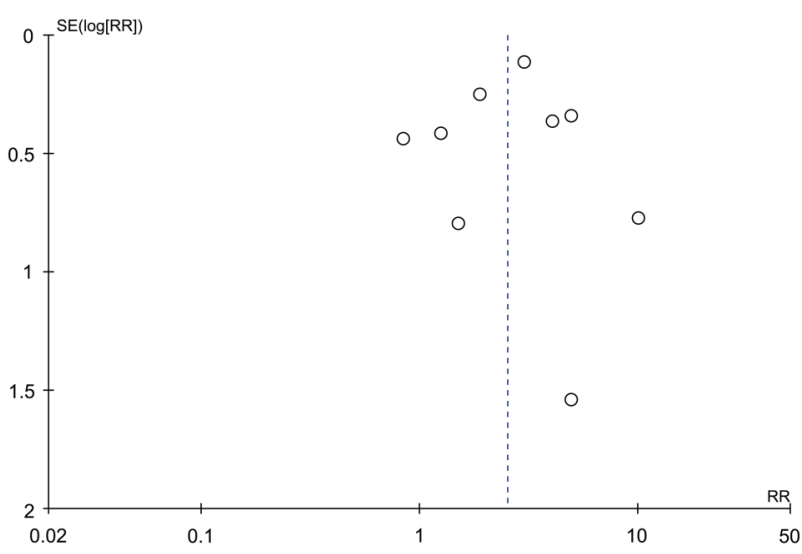

Figure 3 Funnel plot assessing potential for publication bias within anaesthetic outcome studies included in meta-analysis.

The subgroup of grouped RAE studies only was marginally non-significant with a risk ratio of 1.75 (95\% CI 0.95 to 3.21 , $\mathrm{p}=0.07)$. The subgroup of only the studies that achieved a high-quality score on the Newcastle-Ottawa scale retained a significant risk ratio of $2.38 \quad(95 \%$ CI 1.45 to 3.90 , $\mathrm{p}<0.0001)$.
No evidence of publication bias was identified when examined through a funnel plot (figure 3).

Sensitivity analysis showed that significance was retained if any individual study was removed, and also if the two most heavily weighted studies were removed.

\section{Surgical outcomes}

Table 3 shows that there was inconsistency between studies on the impact of ETS exposure on prognosis after ENT surgical procedures. Three studies ${ }^{32} 3839$ showed no significant effects on outcomes, although two studies suggested some evidence that ETS exposure decreased the effectiveness or duration of tympanostomy (ventilation) tubes. ${ }^{28} 35$ Other studies showed ETS exposure caused a potential deterioration of either subjective $^{293436}$ or objective 30313340 outcomes.

\section{Secondary outcomes}

One paper ${ }^{37}$ considered the impact of surgery on smoking behaviours for relatives of patients, a secondary outcome. Their analysis of survey data showed that parents who smoked were more likely to make a cessation attempt if their child had had recent surgery (OR 2.61 CI 95\% 1.56 to $4.35, \mathrm{p}<0.001$ ), but that they were not more likely to maintain abstinence (OR $0.51 \mathrm{CI}$ $95 \% 0.20$ to $1.28, \mathrm{p}=0.150)$. 
Table 3 Key surgical outcomes relevant to the protocol

\begin{tabular}{|c|c|}
\hline Study & Key findings \\
\hline Atef et $a l^{30}$ & $\begin{array}{l}\text { Mean recovery of postoperative area was } \\
\text { significantly lower in those exposed to ETS } \\
\text { compared with those who were not }(p<0.001) \text {. }\end{array}$ \\
\hline Chen et $a l^{29}$ & $\begin{array}{l}\text { Children resident in households with smokers were } \\
\text { at higher risk of cough following previous tonsil/ } \\
\text { adenoidectomy }(p<0.001) \text {. }\end{array}$ \\
\hline Gov-Ari et $a l^{38}$ & $\begin{array}{l}\text { Smoke exposure was not a significant predictor } \\
\text { variable for requiring tonsillectomy after previous } \\
\text { adenoidectomy. }\end{array}$ \\
\hline $\begin{array}{l}\text { Hammarén-Malmi } \\
\text { et } a l^{28}\end{array}$ & $\begin{array}{l}\text { Once confounding factors were controlled for, only } \\
\text { maternal smoking increased risk of recurrent acute } \\
\text { otitis media (OR } 4.1595 \% \mathrm{Cl} 1.45 \text { to 11.9) after } \\
\text { tympanostomy tube insertion. }\end{array}$ \\
\hline Ilicali et $a l^{39}$ & $\begin{array}{l}\text { No significant difference in early ventilation tube } \\
\text { extrusion or persistent otorrhea due to passive } \\
\text { smoking demonstrated (maternal smoking OR } 0.69 \\
\text { Cl } 95 \% 0.29 \text { to 1.67). }\end{array}$ \\
\hline Ilicali et $a l^{32}$ & $\begin{array}{l}\text { No significant difference in postoperative } \\
\text { complication rates due to ETS exposure. }\end{array}$ \\
\hline Kim et $a l^{40}$ & $\begin{array}{l}\text { Indirect smoking not significantly associated with } \\
\text { being in poor outcome group. } \\
\text { Multivariate model showed indirect smoking as } \\
\text { marginally significant factor (OR } 3.377 \mathrm{CI} 95 \% \\
1.032 \text { to 11.053). }\end{array}$ \\
\hline Maw and Bawden ${ }^{31}$ & $\begin{array}{l}\text { Hazard rate for resolution in ears exposed to } \\
\text { parental smoke, } 0.61(\mathrm{Cl} 95 \% 0.47 \text { to } 0.79) \text {. } \\
\text { Parental smoking detrimental to clearance of fluid } \\
\text { in Cox regression analysis. }\end{array}$ \\
\hline Praveen and Terry ${ }^{35}$ & $\begin{array}{l}\text { Lower median survival of grommet if both parents } \\
\text { smoke }(59.9 \text { weeks } \mathrm{Cl} 95 \% 53.9 \text { to } 65.9) \text { vs neither } \\
\text { parent smokes ( } 86.0 \text { weeks } \mathrm{Cl} 95 \% 78.7 \text { to } 93.3) \text {. } \\
\text { Increased infection rate for passive smoke exposure } \\
\text { ( } \mathrm{p}<0.001) \text {. } \\
\text { Increased myringosclerosis for passive smoke } \\
\text { exposure ( } 64 \% \text { vs } 20 \%, p<0.001 \text { ). }\end{array}$ \\
\hline Ramadan $^{33}$ & $\begin{array}{l}\text { Rate of second look abnormal findings in no steroid } \\
\text { group, } 100 \% \text { in ETS exposed vs } 65 \% \text { in non-ETS } \\
\text { exposed; and } 43 \% \text { in ETS exposed vs } 25 \% \text { in } \\
\text { non-ETS exposed for steroid-treated group. No } \\
\text { statistics calculated. }\end{array}$ \\
\hline Ramadan $^{34}$ & $\begin{array}{l}\text { Univariate analysis showed no significant difference } \\
\text { in success rate due to smoke exposure. Multivariate } \\
\text { analysis reported cigarette smoke exposure as } \\
\text { predictor of success (no statistics presented). }\end{array}$ \\
\hline $\begin{array}{l}\text { Ramadan and } \\
\text { Hinerman }^{36}\end{array}$ & $\begin{array}{l}\text { Endoscopic sinus surgery less successful in ETS } \\
\text { exposed ( } 70 \% \text { vs } 90 \%, \mathrm{p}=0.007 \text { ). } \\
\text { Multivariate analysis showed smoke exposure a } \\
\text { significant factor (OR } 3.795 \% \mathrm{Cl} 1.2 \text { to } 11.3 \text { ). }\end{array}$ \\
\hline
\end{tabular}

\section{DISCUSSION}

\section{Summary of evidence}

The range of papers, generally assessed as high quality, show that ETS exposure increases the risk of anaesthetic complications for children undergoing surgery. The pooled estimate suggests that children are two and a half times more likely to experience a respiratory adverse event. Subgroup analysis suggests laryngospasm is a particular risk for exposed children.

No evidence was identified considering whether there were delayed harms such as infection or delayed wound and bone healing. The only evidence of the impact on surgical outcomes relates to ENT surgery, with a mixed picture of impact on surgical success. The quality of studies is mixed, and complex interactions make establishing causal linkages difficult.
Only one observational study ${ }^{37}$ looked at the impact of surgery on parental behaviour. It showed parents were more likely to make a quit attempt if their child had surgery in the preceding year, but that this attempt was no more likely to be successful.

The review is important to clinical teams in anticipating the increased risks of complications and discussing anaesthesia risks for ETS-exposed children. This information may create a 'teachable moment' for parents and carers to consider smoking cessation, giving our findings public health implications as well.

\section{Strengths and limitations}

This review is the first to bring together evidence of the impact of ETS exposure on outcomes of paediatric surgery. The search strategy was comprehensive and applied across the appropriate range of sources. It is disappointing that it did not identify papers covering outcomes such as infection and wound healing, but this is judged to be due to an absence of evidence.

Papers relating to anaesthetic outcomes were of good quality, reflecting the short-term outcomes being observed, and opportunities to blind observers to exposure status. A variety of methods were used for assessing ETS levels, but biological markers offered more robust techniques for stratifying cohorts. Where less reliable methods were used alongside biological markers, it was reassuring to note reasonable segregation of the cohorts.

The surgical outcomes evidence is limited. ETS exposure was often considered as a coincidental variable or subgroup. This was accommodated by evaluating paper quality solely on the interaction within the ETS study element, but it meant papers scored poorly on quality assessment because they had never been conceived to address our research question.

Also, ETS exposure increases the incidence and prevalence of ENT conditions, and we were clear that an appropriate temporal relationship was required; the detail available in some studies meant a reverse relationship could not be excluded. This is illustrated by Chen's ${ }^{29}$ conclusion that tonsillectomy increased the influence of ETS on cough, as opposed to ETS increasing the persistence of cough after tonsillectomy. Both are feasible interpretations which cannot be separated by this review. Well-conceived methodologies ${ }^{28} 32$ that can demonstrate temporality do exist that can address this.

\section{Conclusions}

Exposure to ETS increases the risk of respiratory adverse events during anaesthesia for children undergoing surgery. It may also impact on the success of surgical procedures, particularly in ENT surgery. Exploration is now needed of whether information on increased risk provides an opportunity to promote smoking cessation, and whether even temporary cessation can be effective in reducing risk.

\section{Twitter Follow Christopher Chiswell at @chris_chiswell}

Acknowledgements The authors thank Carole Cummins and Kate Jolly for their advice on the protocol, Carole Cummins for review of the manuscript prior to submission and David Moore for his advice on application of the study quality appraisal tool. Christopher Chiswell is supported by the NIHR CLAHRC West Midlands initiative. This paper presents independent research and the views expressed are those of the author(s) and not necessarily those of the NHS, the NIHR or the Department of Health.

Collaborators Dr Carole Cummins.

Contributors The manuscript has been read and approved by all authors. CC instigated the review. CC and YA drafted the protocol. YA performed the searches. CC and YA screened the results, quality-assessed the papers and extracted data. CC drafted the manuscript for publication and responded to reviewer comments in communication with YA.

Competing interests None declared. 
Provenance and peer review Not commissioned; externally peer reviewed.

Data sharing statement Additional unpublished data from the study (data extraction forms, meta-analysis calculations) are available on request from the corresponding author.

Open Access This is an Open Access article distributed in accordance with the Creative Commons Attribution Non Commercial (CC BY-NC 4.0) license, which permits others to distribute, remix, adapt, build upon this work non-commercially, and license their derivative works on different terms, provided the original work is properly cited and the use is non-commercial. See: http://creativecommons.org/ licenses/by-nc/4.0/

\section{REFERENCES}

1 Jones LL, Hashim A, McKeever T, et al. Parental and household smoking and the increased risk of bronchitis, bronchiolitis and other lower respiratory infections in infancy: systematic review and meta-analysis. Respir Res 2011;12:5.

2 Burke H, Leonardi-Bee J, Hashim A, et al. Prenatal and passive smoke exposure and incidence of asthma and wheeze: systematic review and meta-analysis. Pediatrics 2012;129:735-44.

3 Jones LL, Hassanien A, Cook DG, et al. Parental smoking and the risk of middle ear disease in children: a systematic review and meta-analysis. Arch Pediatr Adolesc Med 2012;166:18-27.

4 Murray RL, Britton J, Leonardi-Bee J. Second hand smoke exposure and the risk of invasive meningococcal disease in children: systematic review and meta-analysis. BMC Public Health 2012;12:1062.

5 (US) Office on Smoking and Health. Respiratory Effects in Children from Exposure to Secondhand Smoke. 2006. http://www.ncbi.nlm.nih.gov/books/NBK44318 (accessed 6 Jan 2016).

6 Royal College of Physicians. Passive smoking and children. A report by the Tobacco Advisory Group. London: RCP, 2010. https://cdn.shopify.com/s/files/1/0924/4392/ files/passive-smoking-and-children.pdf (accessed 6 Jan 2016).

7 Schwilk B, Bothner U, Schraag S, et al. Perioperative respiratory events in smokers and nonsmokers undergoing general anaesthesia. Acta Anaesthesio/ Scand 1997;41:348-55. http://ovidsp.ovid.com/ovidweb.cgi?T=JS\&CSC=Y\&NEWS=N\& PAGE $=$ fulltext\&D=med4\&AN=9113178

8 Sorensen LT. Wound Healing and Infection in Surgery. Arch Surg 2012:147:373.

9 Patel RA, Wilson RF, Patel PA, et al. The effect of smoking on bone healing: a systematic review. Bone Joint Res 2013:2:102-11.

10 Furlong C. Smoking Cessation and its Effects on Outcomes of Surgical Interventions. 2005. http://www.lho.org.uk/viewResource.aspx?id=9078 (accessed 6 Jan 2016)

11 Thomsen T, Villebro N, Møller AM. Interventions for preoperative smoking cessation. Cochrane database Syst Rev 2014;(3):CD002294.

12 Furlong C. Preoperative smoking cessation: a model to estimate potential short term health gain and reductions in length of stay. 2005. http://www.lho.org.uk/ viewResource.aspx?id=9776 (accessed 6 Jan 2016).

13 The Cochrane Collaboration. Review Manager (RevMan). 2014

14 Drongowski RA, Lee $\mathrm{D}$, Reynolds $\mathrm{PI}$, et al. Increased respiratory symptoms following surgery in children exposed to environmental tobacco smoke. Paediatr Anaesth 2003:13:304-10.

15 Jones DT, Bhattacharyya N. Passive smoke exposure as a risk factor for airway complications during outpatient pediatric procedures. Otolaryngol Head Neck Surg 2006;135:12-16.

16 Kim SY, Kim JM, Lee JH, et al. Perioperative respiratory adverse events in children with active upper respiratory tract infection who received general anesthesia through an orotracheal tube and inhalation agents. Korean J Anesthesiol 2013;65:136-41.

17 Lakshmipathy N, Bokesch PM, Cowen DE, et al. Environmental tobacco smoke: a risk factor for pediatric laryngospasm. Anesth Analg 1996;82:724-7.

18 Lyons $B$, Frizelle $H$, Kirby $F$, et al. The effect of passive smoking on the incidence of airway complications in children undergoing general anaesthesia. Anaesthesia 1996:51:324-6.
19 Mamie C, Habre W, Delhumeau C, et al. Incidence and risk factors of perioperative respiratory adverse events in children undergoing elective surgery. Paediatr Anaesth 2004; 14:218-24

20 O'Rourke JM, Kalish LA, McDaniel $\mathrm{S}$, et al. The effects of exposure to environmenta tobacco smoke on pulmonary function in children undergoing anesthesia for minor surgery. Paediatr Anaesth 2006;16:560-7.

21 Reisli R, Apilliogullari S, Reisli I, et al. The effect of environmental tobacco smoke on the dose requirements of rocuronium in children. Paediatr Anaesth 2004;14:247-50.

22 Seyidov TH, Elemen L, Solak M, et al. Passive smoke exposure is associated with perioperative adverse effects in children. J Clin Anesth 2011;23:47-52.

23 Skolnick ET, Vomvolakis MA, Buck KA, et al. Exposure to environmental tobacco smoke and the risk of adverse respiratory events in children receiving general anesthesia. Anesthesiology 1998;88:1144-53.

24 Tait AR, Malviya S, Voepel-Lewis T, et al. Risk factors for perioperative adverse respiratory events in children with upper respiratory tract infections. Anesthesiology 2001;95:299-306

25 Thikkurissy S, Crawford B, Groner J, et al. Effect of passive smoke exposure on general anesthesia for pediatric dental patients. Anesth Prog 2012;59:143-6.

26 Tütüncü AC, Dilmen OK, Utku T, et al. The effects of passive smoking on $\mathrm{COHb}, \mathrm{PaO} 2$ and $\mathrm{PaCO} 2$ levels and postoperative respiratory complications in children undergoing general anesthesia. Turk Pediatr Ars 2012;47:204-9.

27 von Ungern-Sternberg BS, Boda K, Chambers NA, et al. Risk assessment for respiratory complications in paediatric anaesthesia: a prospective cohort study. Lancet 2010;376:773-83.

28 Hammarén-Malmi S, Saxen $\mathrm{H}$, Tarkkanen J, et al. Passive smoking after tympanostomy and risk of recurrent acute otitis media. Int I Pediatr Otorhinolaryngol 2007;71:1305-10.

29 Chen Y, Rennie DC, Lockinger LA, et al. Effect of environmental tobacco smoke on cough in children with a history of tonsillectomy or adenoidectomy. Eur Respir $J$ 1998;11:1319-23.

30 Atef A, Zeid IA, Qotb M, et al. Effect of passive smoking on ciliary regeneration of nasal mucosa after functional endoscopic sinus surgery in children. J Laryngol Otol 2009;123:75-9.

31 Maw R, Bawden R. Spontaneous resolution of severe chronic glue ear in children and the effect of adenoidectomy, tonsillectomy, and insertion of ventilation tubes (grommets). BMJ 1993;306:756-60

32 Ilicali OC, Keles N, De er K, et al. Evaluation of the effect of passive smoking on otitis media in children by an objective method: urinary cotinine analysis. Laryngoscope 2001:111:163-7.

33 Ramadan HH. Corticosteroid therapy during endoscopic sinus surgery in children: is there a need for a second look?. Arch Otolaryngol Head Neck Surg 2001:127:188-92.

34 Ramadan $\mathrm{HH}$. Surgical management of chronic sinusitis in children. Laryngoscope 2004:114:2103-9.

35 Praveen CV, Terry RM. Does passive smoking affect the outcome of grommet insertion in children?. J Laryngol Otol 2005:119:448-54. http://ovidsp.ovid.com/ ovidweb.cgi? T=JS\&CSC $=$ Y\&NEWS=N\&PAGE=fulltext\&D=med5\&AN=15992470

36 Ramadan $\mathrm{HH}$, Hinerman RA. Smoke exposure and outcome of endoscopic sinus surgery in children. Otolaryngol Head Neck Surg 2002;127:546-8.

37 Shi Y, Warner DO. Pediatric surgery and parental smoking behavior. Anesthesiology 2011;115:12-17.

38 Gov-Ari E, Mills JC, Basler KJ. Predictors of tonsillectomy after previous adenoidectomy for upper airway obstruction. Otolaryngol Head Neck Surg 2012;146:647-52.

39 Ilicali OC, Keles N, Değer K, et al. Relationship of passive cigarette smoking to otitis media. Arch Otolaryngol Head Neck Surg 1999;125:758-62.

$40 \mathrm{Kim} \mathrm{HY}$, Dhong HJ, Chung SK, et al. Prognostic factors of pediatric endoscopic sinus surgery. Int J Pediatr Otorhinolaryngol 2005;69:1535-9.

41 Parnis SJ, Barker DS, Van Der Walt JH. Clinical predictors of anaesthetic complications in children with respiratory tract infections. Paediatr Anaesth 2001;11:29-40 